\title{
Evanescent-field optical microscopy: effects of polarization, tip shape and radiative waves
}

\author{
N.F. van Hulst, F.B. Segerink, F. Achten and B. Bölger \\ Opto-electronics, Applied Physics, Unicersity of Twente, P.O. Box 217, 7500 AE Enschede, The Netherlands
}

Received 12 August 1991

\begin{abstract}
Recent results in evanescent-field optical microscopy are presented. A resolution of $30 \mathrm{~nm}$ in the lateral directions and 0.1 $\mathrm{nm}$ in height has been obtained by suitable tip fabrication. Both the direction of the exciting field and the tip shape are shown to affect the optical coupling efficiency and resolution. Near-field diffraction patterns are observed with high lateral resolution by interference between evanescent and propagating waves.
\end{abstract}

\section{Introduction}

The idea to use optical evanescent fields for high-resolution optical microscopy dates back to long before STM [1], yet STM has been essential to the progress of scanning near-field optical microscopy (SNOM) over the last years. Presently optical images with a lateral resolution of $10-100$ $\mathrm{nm}$ have been obtained where the near-field optical probe is either an aperture [2-5] or a dielectric probe tip [6-8], both of nanometer size. the advantage of SNOM compared to STM and AFM is the fact that the optical interaction has a vectorial character and a specific frequency; consequently, the images display polarization contrast and can be spectroscopically analyzed $[9-11]$.

We present improved resolution and new phenomena as observed with our evanescent-field optical microscope [8,12]. In this set-up the optical interaction between a dielectric probe tip and a dielectric sample in close proximity is detected. The system is excited by an evanescent field which, in turn, is generated by total internal reflection (TIR) at a substrate surface supporting the sample. The topographic and dielectric properties of the sample cause a variation in the near field, consisting of both evanescent and homogeneous waves. The sample is imaged in transmis- sion by frustrating the evanescent field with a dielectric probe tip and detecting the fraction that is converted to a homogeneous wave into the probe. The tip is scanned over the sample surface either in constant-height mode, resulting in an image presenting the optical signal variation, or in constant-optical-flux mode ("optical tunneling"), resulting in an image representing topography and variation in the dielectric constant.

Reddick et al. [6] and Courjon et al. [7] have developed a similar set-up, referred to as photon scanning tunneling microscope (PSTM). A theoretical model describing the optical interaction between a dielectric tip and a surface has been presented by Labani [13] and Girard [14].

After a brief description of the working principle and the instrumentation, microscopic results displaying effects of polarization, tip shape and radiative waves are presented and discussed.

\section{Localized frustrated total reflection}

The effect of TIR is well known in optics and generally described by the Fresnel equations. Important for near-field microscopy is the occurrence of a non-radiative exponentially decaying field in the medium beyond the refractive index 


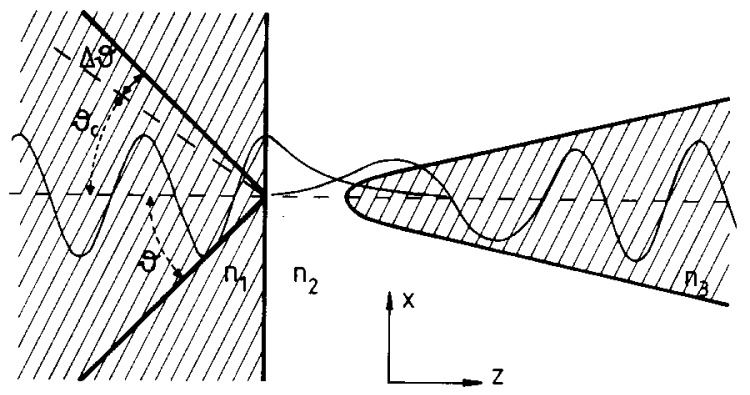

Fig. 1. An evanescent field generated by TIR of a beam incident at a transition from a dense medium $\left(n_{1}\right)$ to a rarer medium $\left(n_{2}<n_{1}\right)$ at an angle $\vartheta$ larger than the critical angle $\vartheta_{c}$ is locally frustrated by a sharp dielectric tip $\left(n_{3}\right)$. The electric field distribution is schematically indicated showing the overlap of the evanescent fields between the plane interface and the probe.

transition, as sketched in fig. 1. This evanescent field is continuous for its component parallel to the surface but discontinuous for its perpendicular component. Consequently, the evanescent field at the surface is generally larger for excitation by p-polarization than for s-polarization.

The presence of a dielectric tip within the evanescent field causes frustrated total reflection (FTR) [15], and part of the light is transmitted into the tip (fig. 1). For an angle of incidence close to the critical angle for TIR the optical transmission is larger for p-polarization than for s-polarization, as can be derived from the complex Fresnel coefficients for TIR [16]. The dependence of the optical transmission on tip-surface separation, angle of incidence and polarization as observed with our set-up [12] is well described by FTR, as was also reported by Reddick [17].

The actual situation of a sharp dielectric probe, with a tip radius appreciably smaller than $\lambda$, and a sample with unknown dielectric and topographic characteristics is more complex. Both non-propagating and propagating waves have to be taken into account. The microscopic treatment of Labani [13] and Girard [14] overcomes this problem. Their model indicates a higher resolution for structures along the direction of the incident field.

\section{Instrumentation}

The dielectric probe tips are fabricated by chemical etching of a single-mode fibre at the meniscus between a HF solution and paraffin oil. The tips have $\sim 20^{\circ}$ apex and $\sim 100 \mathrm{~nm}$ radius. The evanescent field is generated by TIR of a linearly polarized $\mathrm{HeNe}$ laser beam $(\lambda=632.8$ $\mathrm{nm})$ in a glass substrate $(n=1.515)$. The fraction of the laser power coupled into the optical probe by FTR is typically $10^{-7}$, mainly determined by the ratio between the probe tip area and the illuminated sample surface area. Scan pattern generation, data-acquisition and 3D image visualization are controlled by a standard personal computer.

\section{Results and discussion}

Both homogeneous waves propagating in air caused by the presence of a sample and homogeneous waves generated in the fibre by FTR contribute to the detected signal and cannot be distinguished. The FTR contribution is confined to the near-field regime whereas the radiative waves extend into the far field. This is illustrated in fig. 2 , where the probe is scanned over a $1 \mu \mathrm{m}$ period

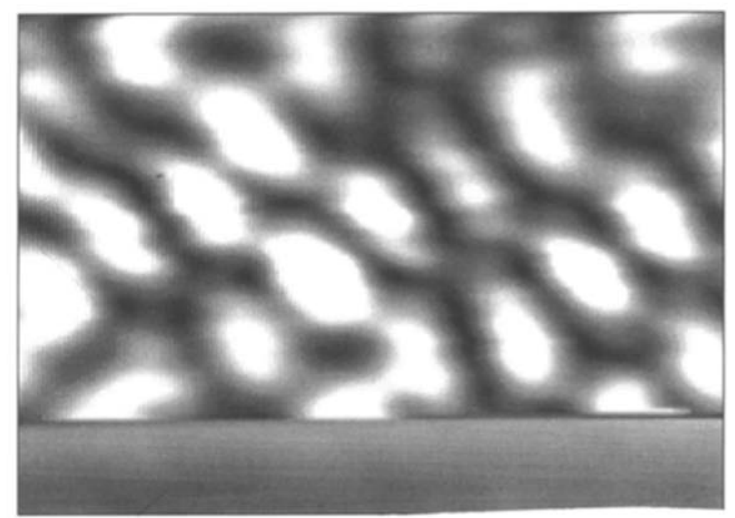

Fig. 2. Field intensity distribution in the plane of incidence above a $1 \mu \mathrm{m}$ period grating with its lines perpendicular to the plane of observation. The p-polarized laser beam is incident from right to left. The image is obtained by continuous line scans over $3 \mu \mathrm{m}$ (horizontal) while approaching the surface over $2 \mu \mathrm{m}$ (vertical) until touch-down. The bottom area of the image displays the signal level while the tip is in contact with the surface. 

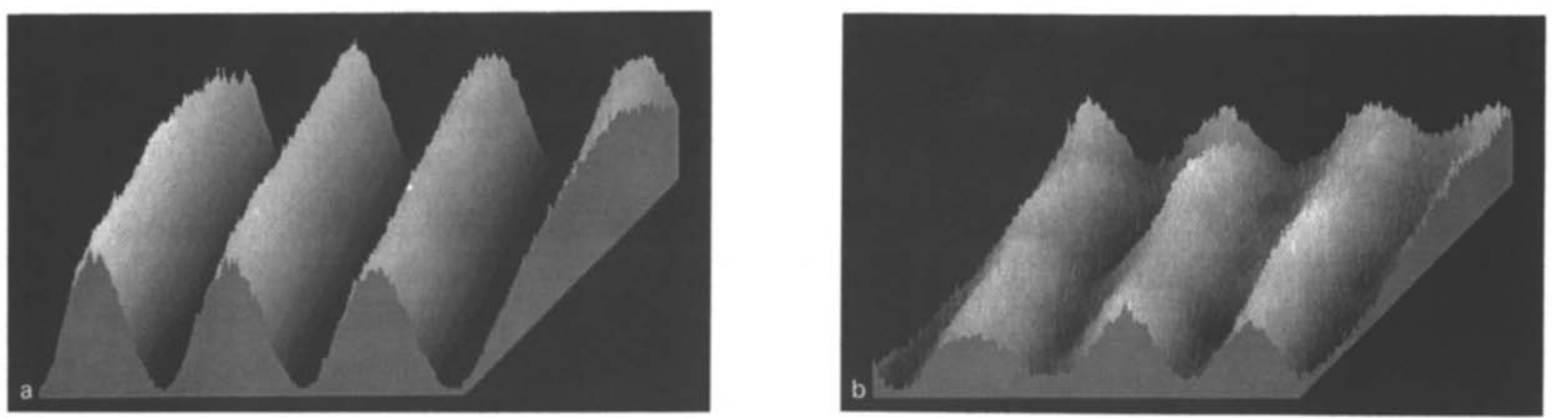

Fig. 3. $3 \times 3 \mu \mathrm{m}$ constant-height scan over a $1 \mu \mathrm{m}$ period SiN grating with a p-polarized (a) and s-polarized (b) laser beam.

grating structure in a plane perpendicular to the surface. The presence of radiative waves extending beyond the near field is obvious. The interference pattern with a period of about the wavelength is generated by radiative waves propagating in different directions and possibly also by stationary waves between tip and surface. Clearly, optical feedback is impossible for the field distribution in fig. 2 . The ratio between the evanescent and radiating field contribution has to be optimized. The direction of the radiative waves depends on the angle of the grating lines relative to the plane of incidence, indicating that they are generated at the edges of the grating lines. For the specific case of a grating, orientation of the lines parallel to the plane of incidence reduces the scattering contribution. Use of a substrate with a higher refractive index, grazing-incidence illumination or a coated fibre could also be of advantage. In any case the characteristic period of the object should be well below the wavelength in order to avoid the disturbing effect of radiative waves.

Fig. 3 shows a constant-height scan in close proximity over the same $1 \mu \mathrm{m}$ period grating for excitation with p-polarization (fig. 3a) and spolarization (fig. 3 b). The periodic field distribution corresponds to the period and direction of the grating structure. The optical signal level for p-polarization excitation is more stable and larger by a factor $\frac{5}{3}$ than the signal for s-polarization. By scanning at a larger distance from the surface we have observed an increasing disturbance of the image by radiatively scattered fields.

Fig. 4 shows scans over a $10 \mu \mathrm{m}$ period photoresist grating structure, with steps $3.6 \mu \mathrm{m}$ wide and $1.5 \mu \mathrm{m}$ high, for p- (fig. 4a) and s-polarization (fig. 4b), both with the same tip in constant-

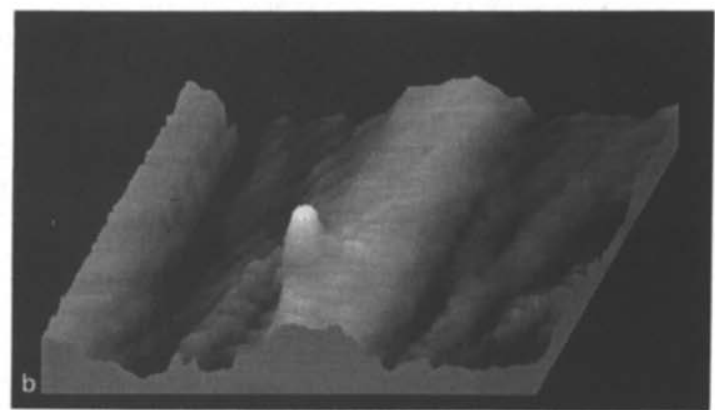

Fig. 4. $18 \times 18 \mu \mathrm{m}$ images of a $10 \mu \mathrm{m}$ period photoresist grating with steps $3.6 \mu \mathrm{m}$ wide and $1.5 \mu \mathrm{m}$ high with a p-polarized (a) and s-polarized (b) laser beam, both in constant-flux mode. 
flux mode. The plane of incidence is parallel to the grating lines. The p-polarization image displays a grating with steps $4.3 \mu \mathrm{m}$ wide and $1.5 \mu$ high, while the s-polarization image only vaguely reveals the grating with $0.9 \mu \mathrm{m}$ step height. Fig. 4a displays steep slopes on the grating edges which are the result of abrupt changes in feedback action. In fig. $4 \mathrm{~b}$ a contamination of size smaller than $1 \mu \mathrm{m}$ is present. Comparison shows that s-polarized excitation tends to couple light in the lateral direction, resulting in both wider and lower steps, whereas p-polarization couples preferentially in the vertical direction.

Tips are fabricated in a standardized procedure, yet their sharpness is rather variable. This is illustrated in fig. 5, showing microscopic images obtained with different tips. The sample is a SiN grating, fabricated by lithographic etching of a peCVD SiN layer, using a $1 \mu \mathrm{m}$ period mask. The grating steps are $500 \mathrm{~nm}$ wide and $350 \mathrm{~nm}$ high. The grating is scanned in the constant-flux mode with s-polarized excitation. In fig. 5a the apparent image is a combination of grating topography and tip shape. The top face of the grating steps displays a smooth modulation pattern with a period of about the wavelength caused by interference of scattered waves. In fig. $5 b$ the tip is so sharp that it follows the grating contour with an average edge width of $30 \mathrm{~nm}$, and topographic structure in the trenches of the grating is resolved. Our observations indicate that the interference pattern becomes less prominent for sharper tips.

An image of a $\varnothing 91 \mathrm{~nm}$ latex sphere, adsorbed to the substrate surface, scanned in constantheight mode is shown in fig. 6a. The s-polarized laser beam is propagating from left to right. The image has been differentiated in the horizontal line scan direction in order to enhance the wave pattern surrounding the sphere. The pattern displays the modulation in field intensity caused by interference between the field scattered by the sphere and the evanescent wave propagating along the surface. The modulation period varies from about $300 \mathrm{~nm}$ for scattering waves counterprogating the incident wave to larger values for other directions. Similar features can be observed in earlier images [17,18]. The $\varnothing 91 \mathrm{~nm}$ latex sphere appears grey with about $500 \mathrm{~nm}$ dimension because its field intensity is more than two orders of magnitude larger than that of the surrounding fields and out of the dynamic range of the detection system.

Fig. 6b displays the near-field diffraction pattern of two $\varnothing 951 \mathrm{~nm}$ latex spheres, scanned in constant-flux mode, with the laser beam propagating from left to right. Due to the close proximity of the tip the spheres have been shifted during the scan. The fringe amplitude corresponds to a height variation of $\sim 50 \mathrm{~nm}$ close to the spheres
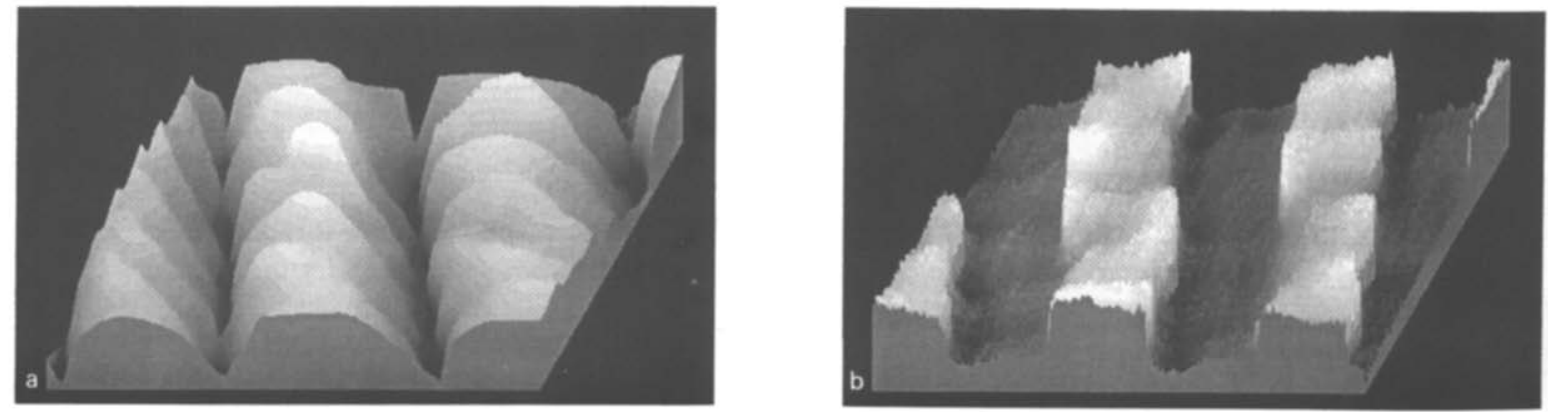

Fig. 5. (a) $3 \times 3 \mu \mathrm{m}$ scan over a $1 \mu \mathrm{m}$ period SiN grating, with steps $500 \mathrm{~nm}$ wide and $350 \mathrm{~nm}$ high, in constant-flux mode with s-polarized laser beam. (b) As (a), but with a sharper tip. 

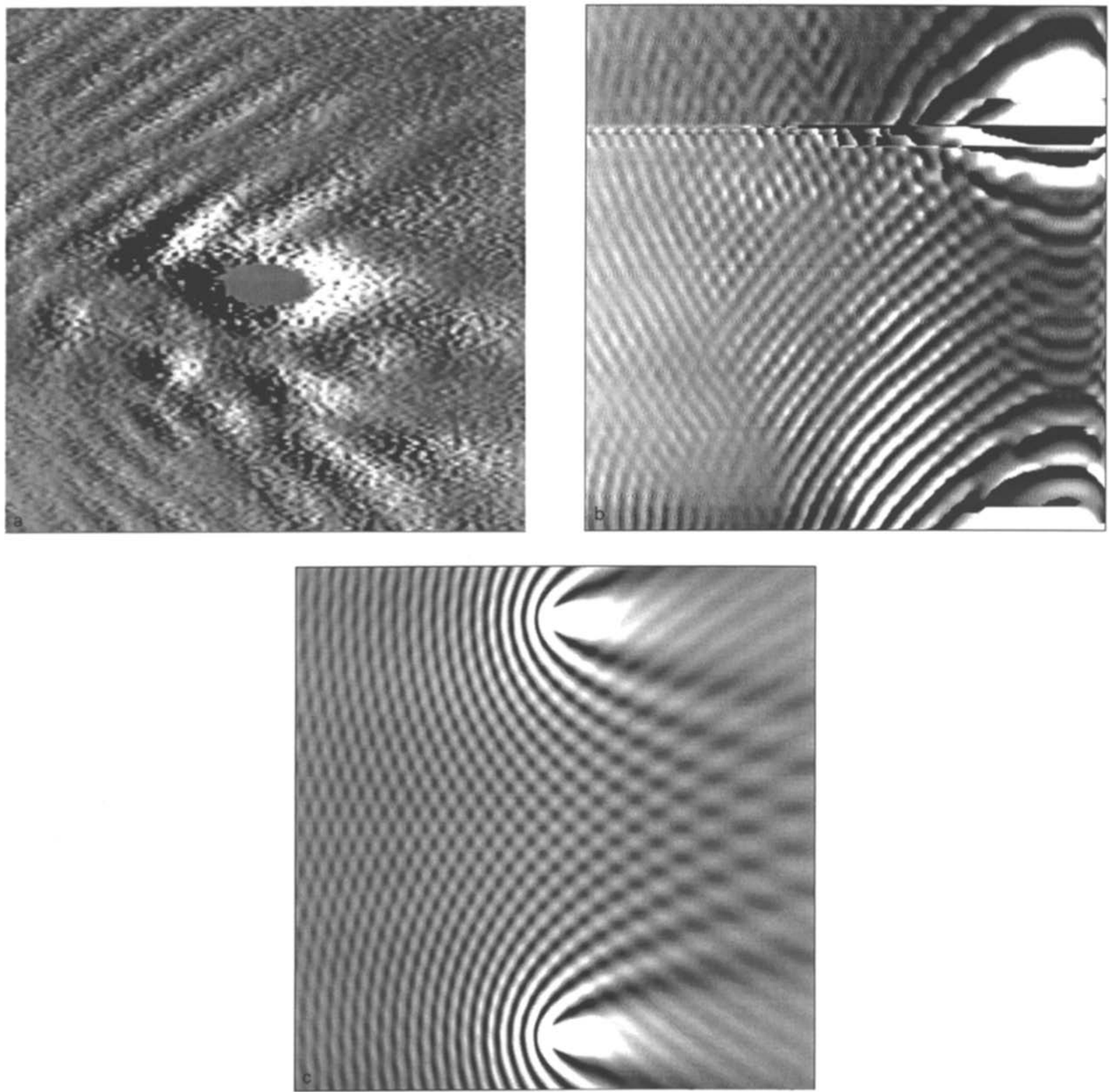

Fig. 6. (a) Differentiated $5 \times 8 \mu \mathrm{m}$ image of a $\varnothing 91 \mathrm{~nm}$ latex sphere with laser beam incident from left to right scanned in constant-height mode. (b) $11.2 \times 11.2 \mu \mathrm{m}$ image of two $\varnothing 951 \mathrm{~nm}$ latex spheres with laser beam incident from left to right in constant-flux mode. (c) Calculated interference pattern $(20 \lambda \times 20 \lambda)$ for two spherical wave sources separated by $16 \lambda$ and a plane wave moving from left to right.

to $\sim 2 \mathrm{~nm}$ at $10 \mu \mathrm{m}$ distance. The fringe period is $320 \pm 10 \mathrm{~nm}$ in the direction antiparallel to the incident wave.

We have calculated the field intensity distribution for two sources of spherical waves 16 wave- lengths apart interfering with a plane wave. The result is plotted in in fig. $6 \mathrm{c}$ with the plane wave propagating from left to right. The correspondence with figs. $6 \mathrm{a}$ and $6 \mathrm{~b}$ is obvious. The fringe period is $\lambda /(1+\cos \phi)$, where $\phi$ is the scatter- 
ing direction relative to the incident beam, in agreement with the observation.

\section{Conclusions}

Our results clearly demonstrate the capacity of evanescent-field optical microscopy to obtain sub-wavelength resolution. The highest resolution is observed in constant-optical-flux mode. The edge resolution of $\sim 30 \mathrm{~nm}$ is mainly limited by the tip shape. The vertical resolution is about 0.1 $\mathrm{nm}$, limited by electronic noise.

Effects of the polarization direction on the apparent image have been observed. The optical coupling occurs preferentially in the direction along the exciting electric field, in agreement with the prediction by Labani [13]. p-Polarization images correspond well to the vertical topographic structure. s-Polarization couples less efficiently and might be more suitable for lateral structures.

The sharpness of the dielectric tip determines the lateral resolution and affects the discrimination between evanescent waves and radiative waves.

Interference between scattered light and the evanescent field allows detailed analysis of nearfield Fresnel diffraction, both in amplitude and phase. On the other hand, the scattered light affects the feedback action in the constant-flux mode and the interference may dominate any topographic fine structure. Therefore, although the classical diffraction limit is surpassed in resolution, still the presence of radiative waves is a fundamental problem in this type of near-field optical microscopy.

\section{References}

[1] J.A. O'Keefe, J. Opt. Soc. Am. 46 (1956) 359.

[2] D.W. Pohl, W. Denk and M. Lanz, Appl. Phys. Lett. 44 (1984) 651;

U. Dürig, D.W. Pohl and F. Rohner, J. Appl. Phys. 59 (1986) 3318.

[3] E. Betzig, M. Isaacson and A. Lewis, Appl. Phys. Lett. 51 (1987) 2088.

[4] U.Ch. Fischer, in: Scanning Tunneling Microscopy and Related Methods, Ed. R.J. Behm (Kluwer, Dordrecht, 1990) pp. 475-496.

[5] E. Betzig, J.K. Trautman, T.D. Harris, J.S. Weiner and R.L. Kostelak, Science 251 (1991) 1468

[6] R.C. Reddick, R.J. Warmack and T.L. Ferrell, Phys. Rev. B 39 (1989) 767.

[7] D. Courjon, K. Sarayeddine and M. Spajer, Opt. Commun. 71 (1989) 23.

[8] N.F. van Hulst, N.P. de Boer and B. Bölger, Trans. Roy. Microsc. Soc. 1 (1990) 239.

[9] U.Ch. Fischer and D.W. Pohl, Phys. Rev. Lett. 62 (1989) 458.

[10] R. Kopelman, A. Lewis and K. Lieberman, J. Lumin. 45 (1990) 289.

[11] M.A. Paesler, P.J. Moyer, C.J. Jahncke, C.E. Johnson, R.C. Reddick, R.J. Warmack and T.L. Ferrell, Phys. Rev. B 42 (1990) 6750.

[12] N.F. van Hulst, N.P. de Boer and B. Bölger, J. Microscopy 163 (1991) 117.

[13] B. Labani, C. Girard, D. Courjon and D. Van Labeke, J. Opt. Soc. Am. B 7 (1990) 936.

[14] C. Girard and D. Courjon, Phys. Rev. B 42 (1990) 9340.

[15] I.N. Court and F.K. von Willisen, Appl. Opt. 3 (1964) 719.

[16] S. Zhu, A.W. Yu, D. Hawley and R. Roy, Am. J. Phys. 54 (1986) 601.

[17] R.C. Reddick, R.J. Warmack, D.W. Chilcott, S.L. Sharp and T.L. Ferrell, Rev. Sci. Instr. 61 (1990) 3669.

[18] N.F. van Hulst, F.B. Segerink and B. Bölger, Opt. Commun. 87 (1992) 212. 